\title{
The inflammatory cell landscape in the lungs of patients with idiopathic pulmonary arterial hypertension
}

\author{
Leigh M. Marsh¹, Katharina Jandt ${ }^{1}$, Gabriele Grünig², Vasile Foris ${ }^{3}$, \\ Mina Bashir ${ }^{1}$, Bahil Ghanim ${ }^{1,4}$, Walter Klepetko ${ }^{4}$, Horst Olschewski ${ }^{1,3}$, \\ Andrea Olschewski ${ }^{1,5}$ and Grazyna Kwapiszewska ${ }^{1,5}$
}

\begin{abstract}
Affiliations: ${ }^{1}$ Ludwig Boltzmann Institute for Lung Vascular Research, Graz, Austria. ${ }^{2}$ Dept of Environmental Medicine and Medicine, NYU School of Medicine, New York, NY, USA. ${ }^{3}$ Division of Pulmonology, Dept of Internal Medicine, Medical University of Graz, Graz, Austria. "Division of Thoracic Surgery, Dept of Surgery, Medical University of Vienna, Vienna, Austria. ${ }^{5}$ Institute of Physiology, Medical University of Graz, Graz,
\end{abstract} Austria.

Correspondence: Leigh M. Marsh, Ludwig Boltzmann Institute for Lung Vascular Research, Stiftingtalstrasse 24, 8010 Graz, Austria. E-mail: Leigh.MarshQlvr.lbg.ac.at

@ERSpublications

Computational flow cytometry details the complex inflammatory cell landscape in patients with pulmonary hypertension http://ow.ly/rjFZ30g1tew

Cite this article as: Marsh LM, Jandl K, Grünig G, et al. The inflammatory cell landscape in the lungs of patients with idiopathic pulmonary arterial hypertension. Eur Respir J 2018; 51: 1701214 [https://doi.org/ 10.1183/13993003.01214-2017].

ABSTRACT Increasing evidence points towards an inflammatory component underlying pulmonary hypertension. However, the conclusive characterisation of multiple inflammatory cell populations in the lung is challenging due to the complexity of marker specificity and tissue inaccessibility. We used an unbiased computational flow cytometry approach to delineate the inflammatory landscape of idiopathic pulmonary arterial hypertension (IPAH) and healthy donor lungs.

Donor and IPAH samples were discriminated clearly using principal component analysis to reduce the multidimensional data obtained from single-cell flow cytometry analysis. In IPAH lungs, the predominant $\mathrm{CD} 45^{+}$cell type switched from neutrophils to $\mathrm{CD}^{+} \mathrm{T}$-cells, with increases in $\mathrm{CD} 4^{+}, \mathrm{CD}^{+}$and $\gamma \delta \mathrm{T}$-cell subsets. Additionally, diversely activated classical myeloid-derived dendritic cells (CD14 ${ }^{-}$HLA$\mathrm{DR}^{+} \mathrm{CD} 11 \mathrm{c}^{+} \mathrm{CD} 1 \mathrm{a}^{+/-}$) and nonclassical plasmacytoid dendritic cells (pDCs; CD14 ${ }^{-} \mathrm{CD} 11 \mathrm{c}^{-} \mathrm{CD} 123^{+} \mathrm{HLA}-$ $\left.\mathrm{DR}^{+}\right)$, together with mast cells and basophils, were more abundant in IPAH samples. We describe, for the first time, the presence and regulation of two cell types in IPAH, $\gamma \delta \mathrm{T}$-cells and pDCs, which link innate and adaptive immunity.

With our high-throughput flow cytometry with multidimensional dataset analysis, we have revealed the interactive interplay between multiple inflammatory cells is a crucial part of their integrative network. The identification of $\gamma \delta \mathrm{T}$-cells and pDCs in this disease potentially provides a missing link between IPAH, autoimmunity and inflammation.

This article has supplementary material available from erj.ersjournals.com

Received: June 192017 | Accepted after revision: Oct 152017

Support statement: This study was supported in part by the Austrian Science Fund (FWF) (P278488-B23) and the Jubilee Foundation of the Austrian National Bank (grant 16187) awarded to G. Kwapiszewska. Funding information for this article has been deposited with the Crossref Funder Registry.

Conflict of interest: Disclosures can be found alongside this article at erj.ersjournals.com

Copyright CERS 2018. This article is open access and distributed under the terms of the Creative Commons Attribution Licence 4.0. 


\section{Introduction}

Pulmonary hypertension $(\mathrm{PH})$ is characterised by severe vascular remodelling, resulting in increased pulmonary vascular resistance. Increasing evidence has pointed towards an inflammatory component being important in the disease development. In $\mathrm{PH}$, elevated numbers of inflammatory cells have been observed in and around remodelled arteries, where the number of infiltrating cells in the perivascular region correlated with intima and media remodelling [1]. Despite the clear link between $\mathrm{PH}$ and dysregulation of the immune system [2], systematic analysis of immune and inflammatory cells in the lungs of $\mathrm{PH}$ patients is still lacking.

It has been long reported that mast cells and B- and T-lymphocytes are increased in patients with diverse forms of $\mathrm{PH}$ [3-7]. Recruited inflammatory cells can release certain mediators that not only directly alter the vessel microenvironment, but also recruit additional circulating inflammatory cells, which in turn further worsens disease progression. The expression of several cytokines and chemokines, such as CCL2, CCL5 or CX3CL1/fractalkine, is increased in lungs and remodelled vessels of PH patients [8-10]. In addition, inflammatory cells are an important source of other mediators, such as danger molecules (HMGB1) and proteases (tryptase), which can lead to enhanced pulmonary smooth muscle cell proliferation when produced locally $[7,11]$.

To date, the analysis of inflammatory cells within the human lung has relied predominantly on immunohistochemical or morphological characterisation $[1,4,9,12]$. However, due to the complexity of marker specificity, the conclusive classification of several inflammatory populations using only a single marker is challenging. Flow cytometry provides a more sophisticated approach to thoroughly identify and quantify different immune cell populations. This technique is well established in the characterisation of cells from human blood or lavage samples, but only a few recent studies have addressed its usefulness in analysing whole tissue samples [13-15]. We have undertaken an unbiased flow cytometry based approach to immunophenotype multiple inflammatory cell populations in the lungs and pulmonary arteries in a large well-characterised group of idiopathic pulmonary arterial hypertension (IPAH) patients and healthy nonutilised donor lung tissue. This is the first study to conduct such a broad analysis to conclusively demonstrate inflammatory components in the pathogenesis of IPAH.

\section{Material and methods}

A detailed description of methods is provided in the online supplementary material.

\section{Lung tissue}

Lung tissues from IPAH patients $(n=16)$ and controls $(n=15)$ were obtained from the Division of Thoracic Surgery, Medical University of Vienna (Vienna, Austria). Nontransplanted donor lung tissue that had been harvested for transplantation, but not implanted because of size-reduced lung transplantation, served as controls $[16,17]$. All lungs were flushed via ante- and retrograde perfusion with Perfadex (XVIVO Perfusion, Göteborg, Sweden) to remove any residual blood. No signs of any inflammatory conditions such as pneumonia, pulmonary tuberculosis or infections with pleural effusions were present. The protocol and tissue usage were approved by the institutional ethics committee (976/2010).

\section{Tissue preparation and flow cytometry}

Two independent samples ( $400 \mathrm{mg}$ each) were taken at random (either peripheral or perihilar) from fresh explant lungs and processed using a standardised protocol in a blinded fashion. Pulmonary arteries were isolated as shown in online supplementary figure S1; briefly, arteries were identified due to their anatomical position and structure, excised and then processed in the same fashion as lung samples. Single-cell suspensions were prepared and stained using four different combinations of cell surface markers as detailed in online supplementary tables S1 and S2 and recorded on a LSRII flow cytometer (BD Biosciences, Vienna, Austria). The results of paired lung samples were then averaged and data presented as percentage of $\mathrm{CD} 45^{+}$cells or number of cells per $\mathrm{mg}$ of tissue.

\section{Biocomputational analysis}

For bioinformatic analysis RStudio (www.rstudio.com) and R (www.r-project.org) was used. Prcomp was used to calculate the principal components from the log-odds ratios of the percentage CD $45^{+}$cells; the first two principal components were plotted using the ggplot2 package. Heatmaps of the log-odds ratios of cell number per tissue weight or percentage $\mathrm{CD} 45^{+}$cells were plotted using the pheatmap package. Nonlinear-dimensionality reduction using t-distributed stochastic neighbour embedding (t-SNE) [18] was performed in FlowJo (Ashland, OR, USA). 


\section{Immunofluorescent staining}

Immunofluorescent staining was performed on paraffin-embedded lung sections as previously described [19]. Confocal images were obtained using a laser-scanning confocal microscope (LSM 510 Meta; Zeiss, Gottingen, Germany).

\section{Statistics}

Statistical analysis was performed in R. Each group was tested for distribution using the Shapiro-Wilk normality test. Statistical significance was determined using the Wilcoxon rank sum test; p-values are indicated on the figures. Scatterplots show boxplot overlays with median and interquartile range.

\section{Results}

\section{Flow cytometric analysis}

In this study, we used multiparameter flow cytometry in combination with bioinformatics analysis to characterise the global inflammatory profile (figure 1) in lungs of patients with IPAH $(n=16)$ and compared it to that of healthy donors $(n=15)$. Demographical and clinical characteristics of patients and donors are described in table 1. Representative images of lung morphology showing classical vascular remodelling in IPAH are shown in online supplementary figure S2.

21 different cell populations were analysed and separated into cells of 1) lymphoid lineage, including Band T-cells, T-cell subpopulations and natural killer (NK) cells and 2) myeloid lineage, consisting of monocytes, macrophages, mast cells, neutrophils and dendritic cells (DCs) among others. In addition, the markers CD1a and HLA-DR (human leukocyte antigen - antigen D related) were used to determine the

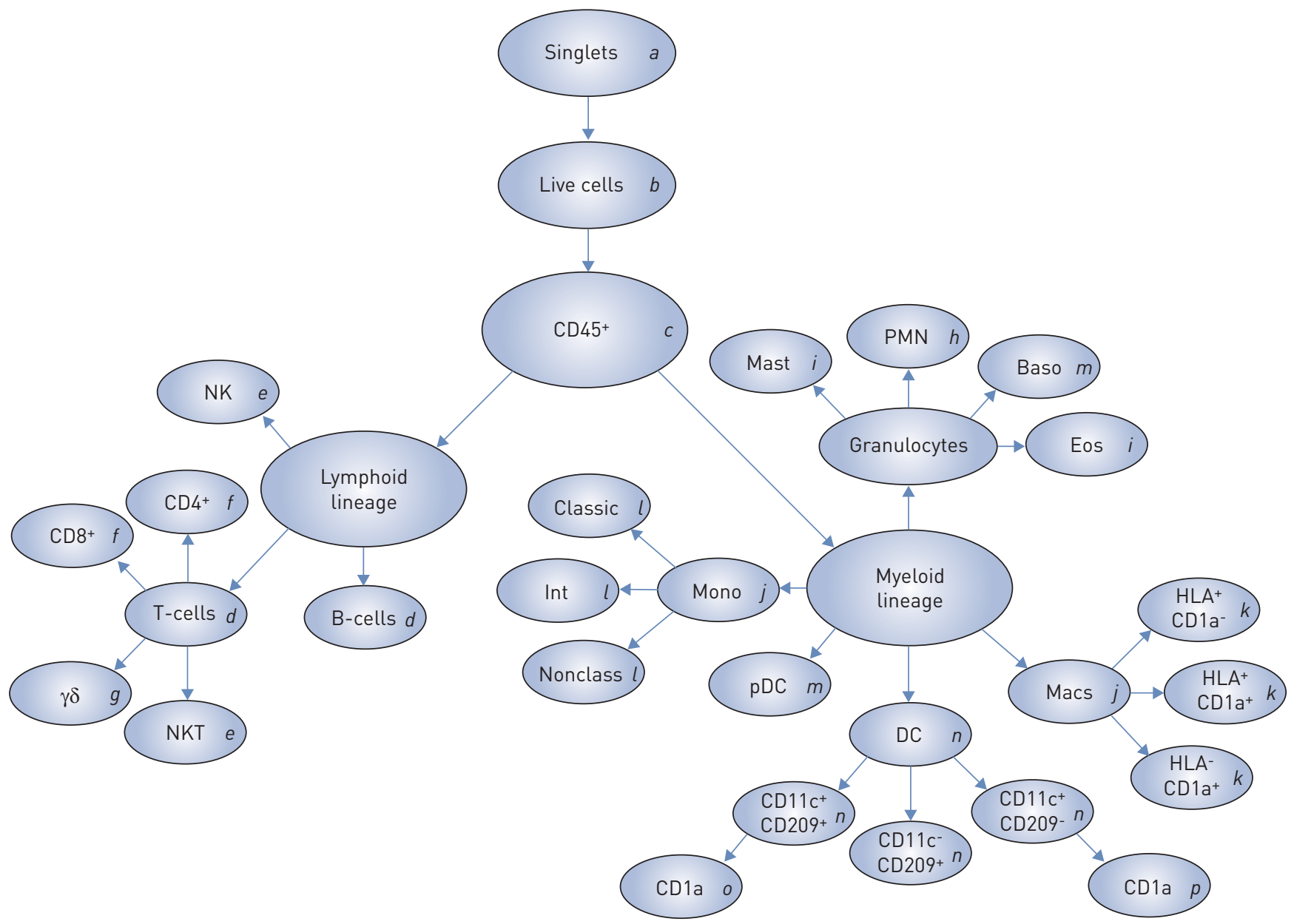

FIGURE 1 Schematic representation of analysed cell populations identified during flow cytometric analysis. Hierarchal organisation of all haematopoietic derived $\mathrm{CD} 45^{+}$cells of both lymphoid and myeloid lineages. NK: natural killer cells; NKT: natural killer T-cells; PMN: polymorphonuclear neutrophils; Baso: basophils; Eos: eosinophils; Mono: monocytes; Int: intermediate monocytes; Nonclass: nonclassical monocytes; DC: dendritic cells; pDC: plasmacytoid DC; Macs: macrophages. Italic letters (a-p) represent common cell populations, as identified in figure 2. 
TABLE 1 Clinical characteristics of the donors and idiopathic pulmonary arterial hypertension (IPAH) patients

\begin{tabular}{|c|c|c|}
\hline & Donors & IPAH patients \\
\hline Lung samples & 15 & 16 \\
\hline Age years & $41.7 \pm 13.2$ & $34.1 \pm 10.6$ \\
\hline Male/female & $10 / 5$ & $5 / 11$ \\
\hline Height $\mathrm{cm}$ & $175 \pm 9$ & $170 \pm 8$ \\
\hline Weight kg & $73.6 \pm 9.9$ & $61.2 \pm 13.3^{*}$ \\
\hline mPAP mmHg & & $71.2 \pm 16.6$ \\
\hline PVR dyn $\cdot \mathrm{sec} \cdot \mathrm{cm}^{-5}$ & & $1845 \pm 669$ \\
\hline 6MWD m & & $280 \pm 187$ \\
\hline NYHA class & & III-IV \\
\hline NT-proBNP pg.mL $\mathrm{m}^{-1}$ & & $4412 \pm 2445$ \\
\hline CRP $\mathrm{mg} \cdot \mathrm{dL}^{-1}$ & & $1.2 \pm 2.4$ \\
\hline \multicolumn{3}{|l|}{ PAH targeted therapy } \\
\hline ERA & & 1 \\
\hline ERA+PDE5i & & 1 \\
\hline $\mathrm{sGCs+PGI}$ & & 1 \\
\hline PDE5i+PGI & & 4 \\
\hline ERA+PDE5i+PGI & & 7 \\
\hline PDE5i+PGI+CCB & & 1 \\
\hline Unknown & & 1 \\
\hline
\end{tabular}

Data are presented as $\mathrm{n}$ or mean \pm SD. mPAP: mean pulmonary arterial pressure; PVR: pulmonary vascular resistance; 6MWD: 6-min walking distance; NYHA: New York Heart Association; NT-proBNP: N-terminal pro-brain natriuretic peptide; CRP: C-reactive protein; ERA: endothelin receptor antagonists; PDE5i: phosphodiesterase type 5 inhibitor; SGCs: soluble guanylate cyclase stimulator; PGI: prostacyclin analogue; CCB: calcium channel blocker. *: $p \leqslant 0.05$.

activation status for DCs and macrophages. Detailed descriptions of the multiple cell surface markers used to describe the cell populations and the comprehensive gating strategy are shown in online supplementary table S2 and figure 2, respectively. Due to the multipanel approach, we first performed correlation analysis to determine how the results varied for cell types identified in the different panels (online supplementary figure S3). A strong correlation was observed between panels, which demonstrated the robustness of our analysis and enabled individual values to be averaged, thereby allowing us to generate the largest possible coverage of cell populations for subsequent analysis. Similarly, the comparison of the flow cytometric results obtained from the two independent samples revealed high reproducibility (online supplementary figure S4).

\section{Global changes in inflammatory profiles}

Next, we sought to determine whether IPAH and healthy control lungs show distinct immune cell signatures. To this end, we made use of the latest developments in computational flow cytometry [20] and performed principal component analysis (PCA) to dimensionally reduce the complete dataset while maximising the variance between donors and IPAH samples. Using only lungs with a complete dataset, thereby analysing all 21 cell populations in a total of seven donor and five IPAH lungs gave a good separation of donor and IPAH samples (figure 3a). We then enhanced the power of the PCA by omitting the limiting three cell populations (eosinophils, NK, NKT-cells) from the global analysis. This procedure maximised both the number of analysed lungs and populations and gave a superior discrimination between groups (figure 3b). No differences were observed in the relative levels of these three cell populations between donors and IPAH (online supplementary figure S5). This final dataset, comprising 31 lungs and 18 cell populations was analysed in depth.

\section{Cell distribution of $\mathrm{CD} 45^{+}$cells in the lungs of IPAH patients and donors}

In control samples, $\sim 64 \%$ of all $\mathrm{CD} 5^{+}$cells were from the myeloid lineage, predominantly granulocytes $(32 \%)$ and monocytes (17\%); and $\sim 15 \%$ of the lymphoid lineage, primarily T-cells (14\%), with almost equal amounts of $\mathrm{CD}^{+}$and $\mathrm{CD}^{+}$T-cells. Minor populations included B-cells, basophils, DCs, $\gamma \delta \mathrm{T}$-cells and mast cells (figure 4a). In IPAH lungs, the distribution of immune cells was distinctive, supporting the clear clustering visible in the PCA plots. The strong predominance of myeloid cells was reduced to 52\%, while lymphoid-derived cells were enriched to $31 \%$. Thus, the largest single cell classification in IPAH lungs switched to T-cells (29\%), which corresponded to a clear relative decrease in granulocytic 

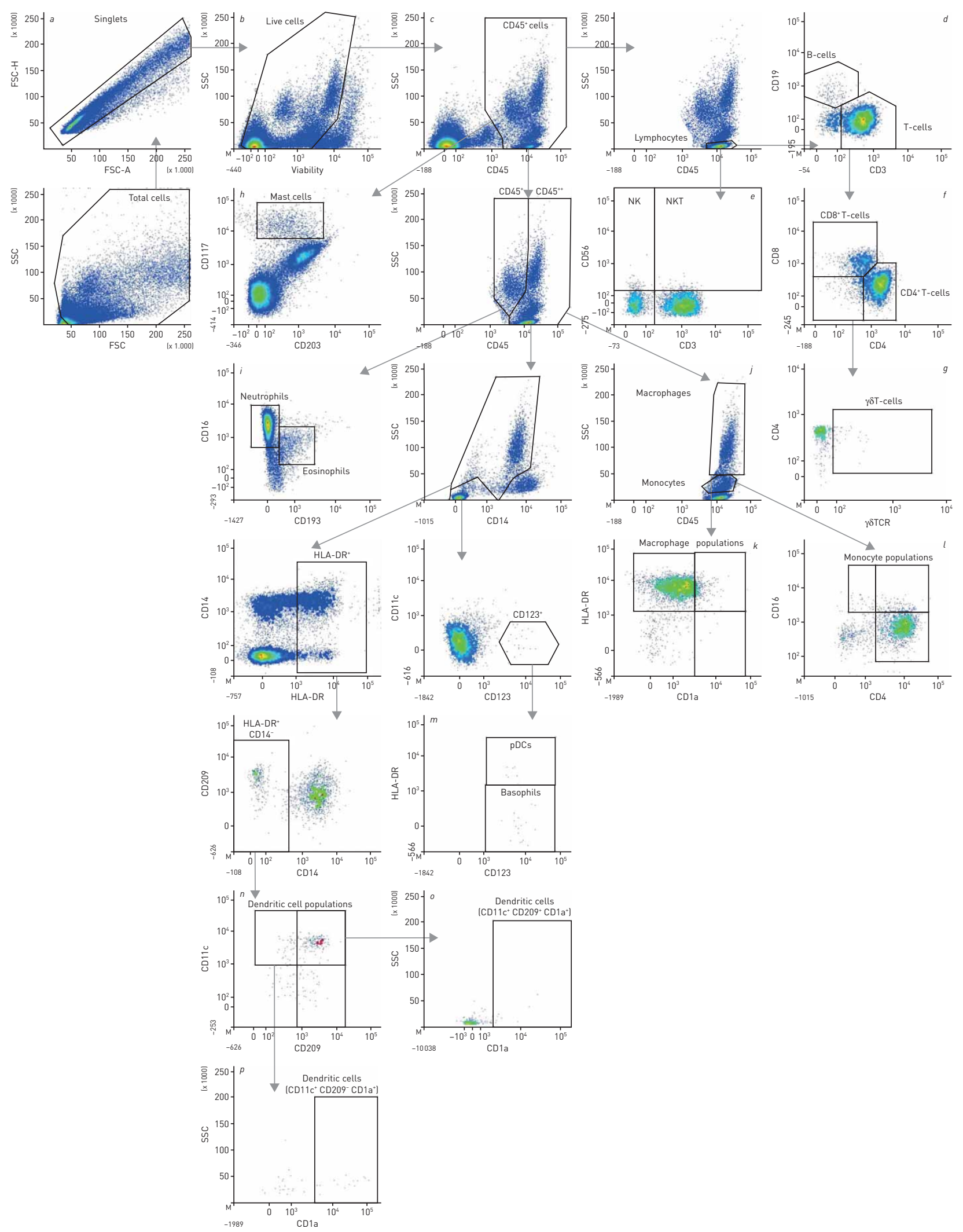

FIGURE 2 Overview of gating strategy used in flow cytometric analysis. Analysed populations were first gated to remove cell clumps and debris, and dead cells were removed by viability exclusion. All leukocytes were selected using CD45-positivity and then divided into separate cell populations as shown. FSC-H: forward scatter height; FSC-A: forward scatter area; SSC: side scatter; HLA-DR: human leukocyte antigen antigen D related; pDCs: plasmacytoid dendritic cells; NK: natural killer cells; NKT: natural killer T-cells. Boxes and italic lettering (a-p) represent common cell populations, as identified in figure 1. 

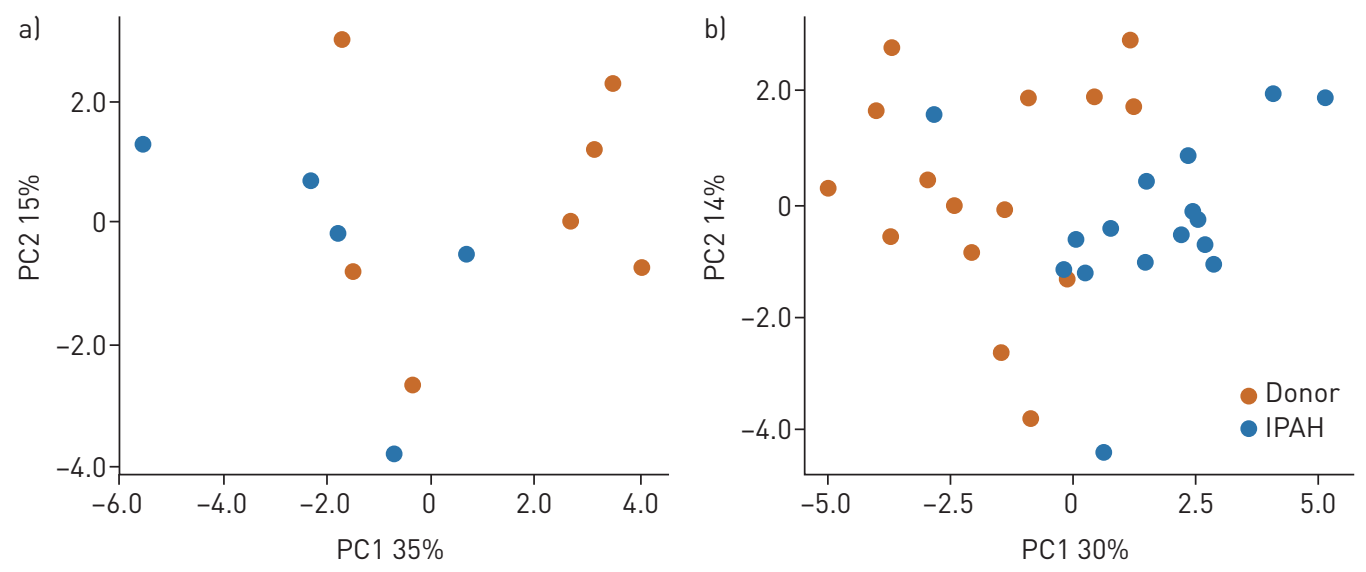

FIGURE 3 Principal component analysis plots of flow cytometric data separates idiopathic pulmonary arterial hypertension (IPAH) from control samples. The log odds ratios of subset analysis data obtained by flow cytometry using manual gating were used to construct a similarity matrix for the principal component analysis. The first two principal components (PC) with respective percentage variation are depicted. Bivariate plots of dimensionality reduction from a) 12 lungs (seven donor/five IPAH) including 21 cell populations; b) 31 lungs (15 donor/16 IPAH) and 18 cell populations.

populations (19\%) as well as myeloid-derived monocytic populations (11\%). In addition, a higher relative abundance of several minor populations including DCs and mast cells was observed (figure 4a). Approximately $21 \%$ of cells in control samples and $16 \%$ in IPAH could not be conclusively identified with the markers used.

Using heatmap representations we examined how the distribution of the percentage CD45 ${ }^{+}$cells (online supplementary figure S6) and the absolute numbers (figure 4b) of each cell type varied in every patient. Within each group, relatively homogeneous patterns were observed, while between IPAH and donor

a)
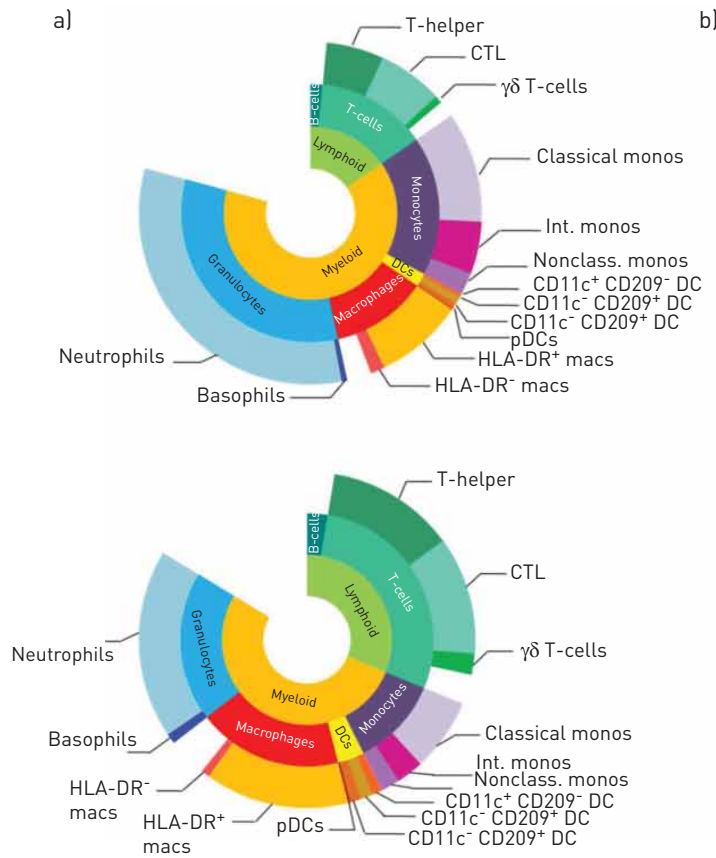

b)

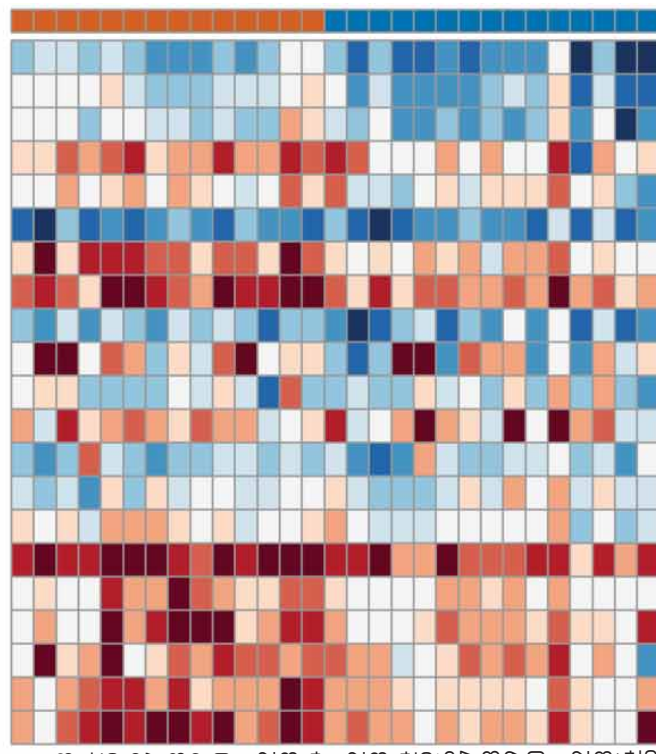

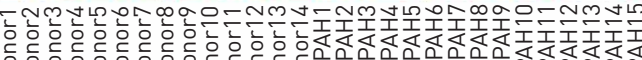

Sample

T-cells

$\mathrm{CD} 4^{+} \mathrm{T}$-cells

CD8 ${ }^{+} \mathrm{T}$-cells

$\gamma \delta$ T-cells

B-cells

PMN

Mast

Basophils

Macrophages

Macs (HLA+ CD1a-)

Macs (HLA+ $\left.\mathrm{CD}^{+} \mathrm{a}^{+}\right)$

Macs (HLA- CD1a+)

Monos (classical)

Monos (intermediate)

Monos (nonclassical)

pDCs

DCs (CD11 $\mathrm{C}^{+}$CD209+)

DS (CD11 c+ CD209+ CD1a+)

DCs (CD11 c- CD209+)

DCs (CD11c+ CD209-)

$\mathrm{DCs}\left(\mathrm{CD} 11 \mathrm{c}^{+} \mathrm{CD} 209^{-} \mathrm{CD} 1 \mathrm{a}^{+}\right)$

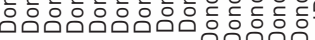

FIGURE 4 Global changes in inflammatory profiles. a) Sunburst plots showing average distribution of all cell populations in donor lungs and lungs from patients with idiopathic pulmonary arterial hypertension; b) heatmap representation presenting the patient-to-patient variability of the log

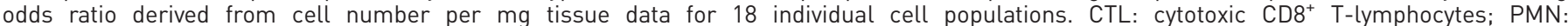
polymorphonuclear neutrophils; Macs: macrophages; Monos: monocytes; Int: intermediate; Nonclass: nonclassical; DCs: dendritic cells; pDCs: plasmacytoid DCs. 

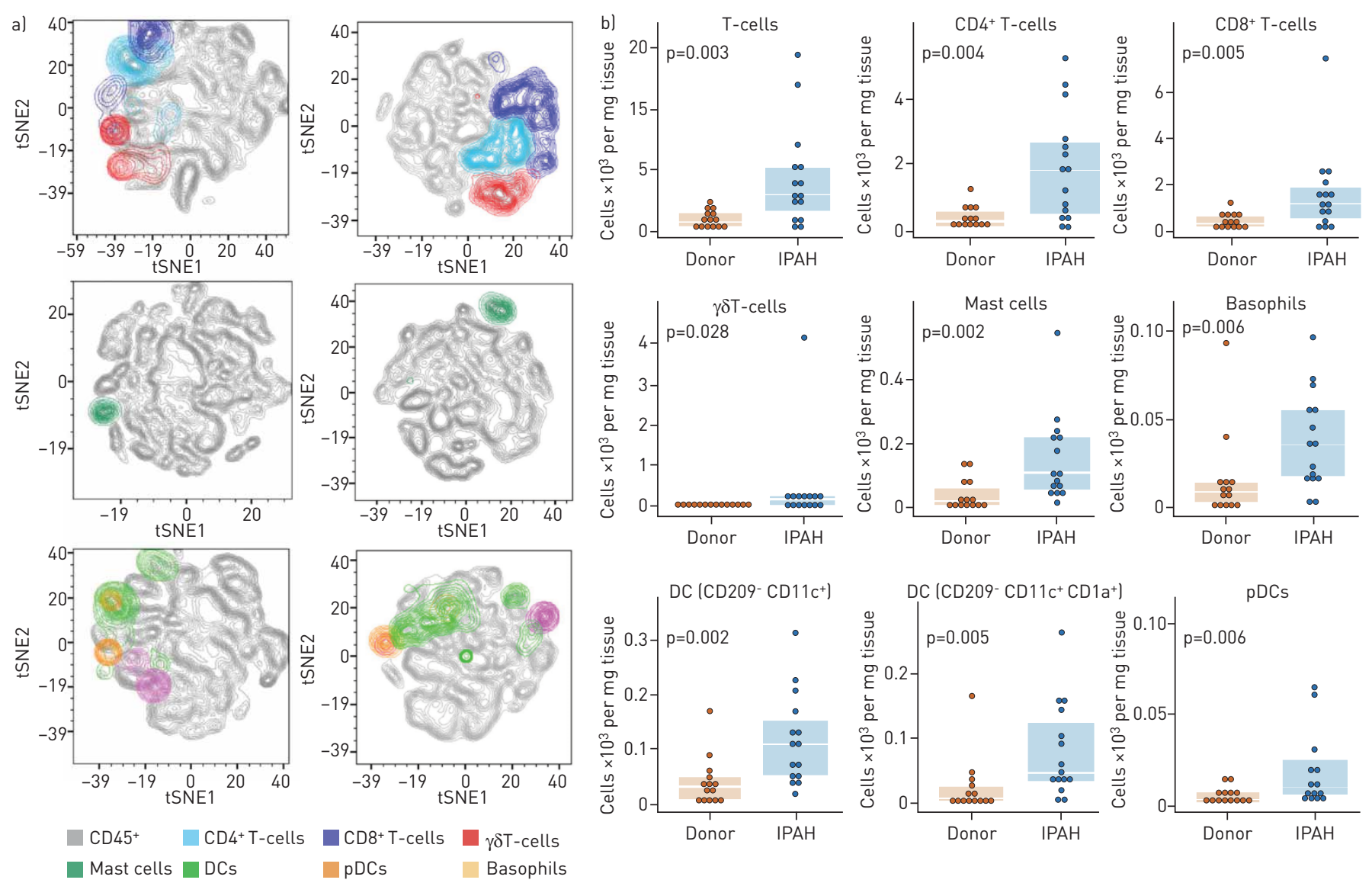

FIGURE 5 T-stochastic neighbour embedding (t-SNE) visualisation and regulated cell populations in lung samples. a) t-SNE composite dimension plots of down-sampled and concatenated $\mathrm{CD}_{4} 5^{+}$cells derived from flow cytometric data with overlaid manually gated cell populations. b) Regulated cell populations differentially abundant between idiopathic pulmonary arterial hypertension and donor lungs as identified by flow cytometry. DCs: dendritic cells; pDCs: plasmacytoid dendritic cells. Boxplots show median and interquartile range.

samples, well-defined differences were found, as highlighted by the enrichment of T-cell and DC populations in IPAH patients (figure 4b). Using Euclidean distance calculation to order the samples resulted in good clustering of IPAH and donor samples (online supplementary figure S7).

\section{Regulation of individual cell types in lung samples}

Moving from global differences to the changes in individual cell types, we analysed the 18 cell populations represented in figure $4 \mathrm{~b}$ individually. When analysing cell numbers, 10 cell populations were differentially abundant between IPAH and donor lungs (figure 5). These populations (as identified in figure 2) were then mapped on t-SNE composite plots, which revealed a clear discrimination and localisation of most populations (figure 5a). t-SNE plots highlight the relationship between different cell clusters on a new bivariate matrix obtained by taking into account the similarities of all original dimensions. The altered inflammatory state in IPAH lungs is demonstrated by an increased number of T-cells $\left(\mathrm{CD} 4^{+}\right.$and $\mathrm{CD} 8^{+}$ T-cells), mast cells, basophils and DCs. In addition, we identified elevated levels of $\gamma \delta \mathrm{T}$-cells, a population which has not been reported in PH, so far. Within the myeloid-derived CD11c $\mathrm{c}^{+} \mathrm{DC}$ group, both $\mathrm{CD}^{2} 09^{+}$ and $\mathrm{CD}_{209^{-}}$subpopulations were increased, including $\mathrm{CD}^{+} \mathrm{a}^{+}$activated DCs (figure $5 \mathrm{~b}$ and online supplementary figure S7). Of note, the DC overlay in figure 5a shows a more dispersed population in the IPAH sample compared to donors. This can arise from the multiple DC populations being regulated and activated in IPAH lungs. In addition, the nonmyeloid dendritic cells, pDCs, were increased in the IPAH lungs. No changes were observed in the absolute abundance of $\mathrm{CD}_{1} 9^{+} \mathrm{B}$-cells, macrophage subpopulations (differing in their expression of HLA-DR and CD1a), $\mathrm{CD} 11 \mathrm{c}^{-} \mathrm{CD} 209^{+} \mathrm{DCs}$, monocyte subpopulations or neutrophils (online supplementary figure S8). Closer examination of the percentage of $\mathrm{CD} 45^{+}$cells revealed similar regulation in the majority of cell types (online supplementary figure S9). However, some populations, such as neutrophils and monocytes, were decreased, which probably arises from the strong increase in T-cell populations (online supplementary figure S9). 
a)

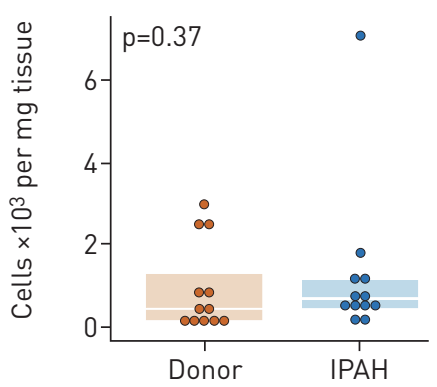

d)

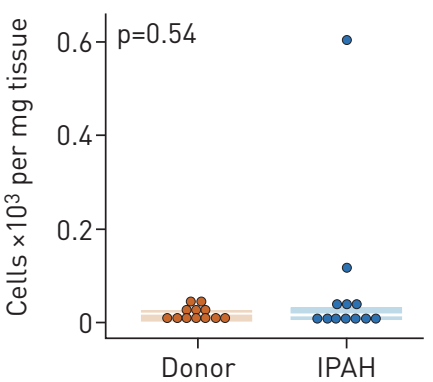

g)

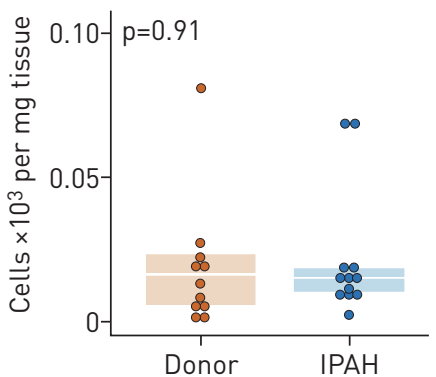

b)

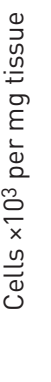

e)

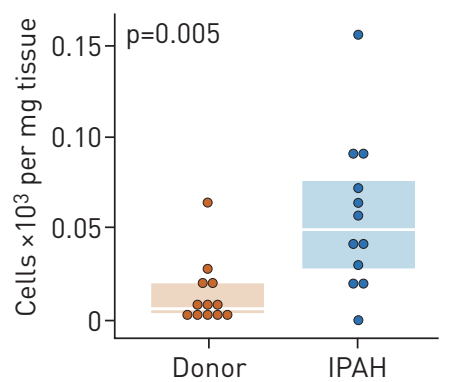

h)

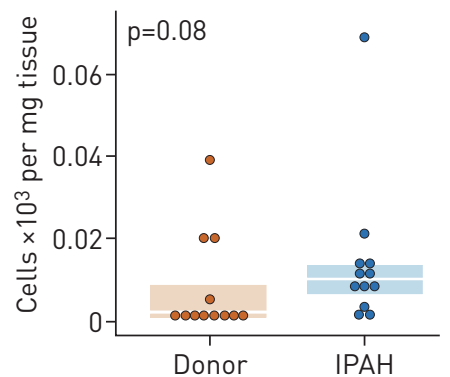

c)

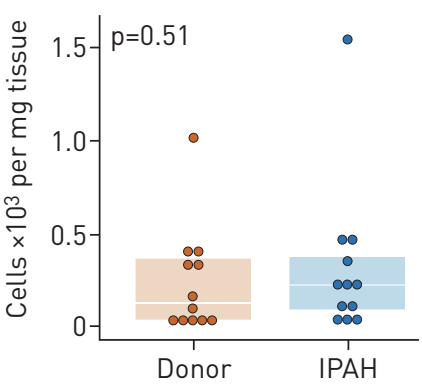

f)

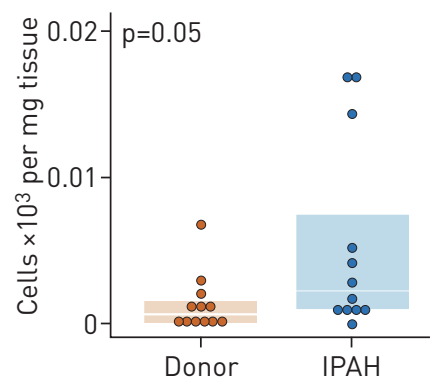

i)

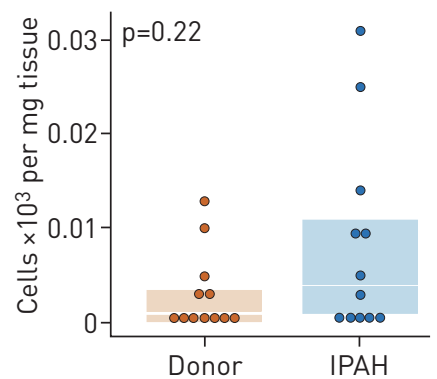

FIGURE 6 Abundance of cell populations in isolated pulmonary arteries. The presence of multiple cell populations (as shown for lung homogenate samples) measured in third-and fourth-generation pulmonary arteries by flow cytometry. a) T-cells; b) $\mathrm{CD}^{+} \mathrm{T}$-cells; c) CD8 ${ }^{+} \mathrm{T}$-cells; d) $\gamma \delta \mathrm{T}$-cells; el mast cells;

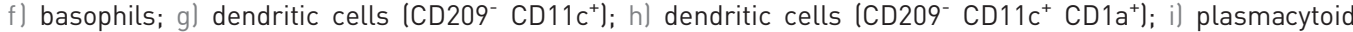
dendritic cells. IPAH: idiopathic pulmonary arterial hypertension. Boxplots show median and interquartile range.

Regulation of inflammatory profiles in isolated pulmonary arteries

We examined whether this cell regulation is restricted to lung tissues that contain multiple compartments, such as small pulmonary arteries, or if it can be found within larger pulmonary arteries. Indeed, even in these large vessels (third to fourth generation; online supplementary figure S1), we observed similar trends with increased numbers of mast cells and basophils; however, several cell types were below statistical significance (figure 6). In addition, we observed increased abundance of activated macrophages $\left(\mathrm{CD}_{\mathrm{a}}{ }^{+} \mathrm{HLA}^{+}\right)$and DCs $\left(\mathrm{CD} 11^{+} \mathrm{CD} 209^{+} \mathrm{CD}^{+} \mathrm{a}^{+}\right)$and monocyte populations (online supplementary figure S10).

Localisation of $p D C s$ and $\gamma \delta T$-cells

As both pDCs and $\gamma \delta \mathrm{T}$-cells are cell types that have previously not been examined in $\mathrm{PH}$, we additionally performed multicolour immunofluorescent staining to confirm and visualise their presence in the lung tissue. pDCs $\left(\mathrm{CD} 123^{+} \mathrm{CD} 304^{+}\right)$were found with increased prevalence in lungs of IPAH patients compared to donors and were localised predominantly in the alveolar space in proximity to vessels (figure $7 \mathrm{a}$ and $\mathrm{b}$ ). $\gamma \delta \mathrm{T}$-cells could be detected in the lungs of both donor and IPAH patients, and in close proximity to arteries in the latter with higher frequency (figure $7 \mathrm{c}$ and $\mathrm{d}$ ).

\section{Circulating $p D C$ levels}

Finally, we investigated whether the increased pDC numbers, as observed in IPAH lungs, could also be detected in the circulation. The characteristics of the outpatient $\mathrm{PAH}$ group and controls are given in 

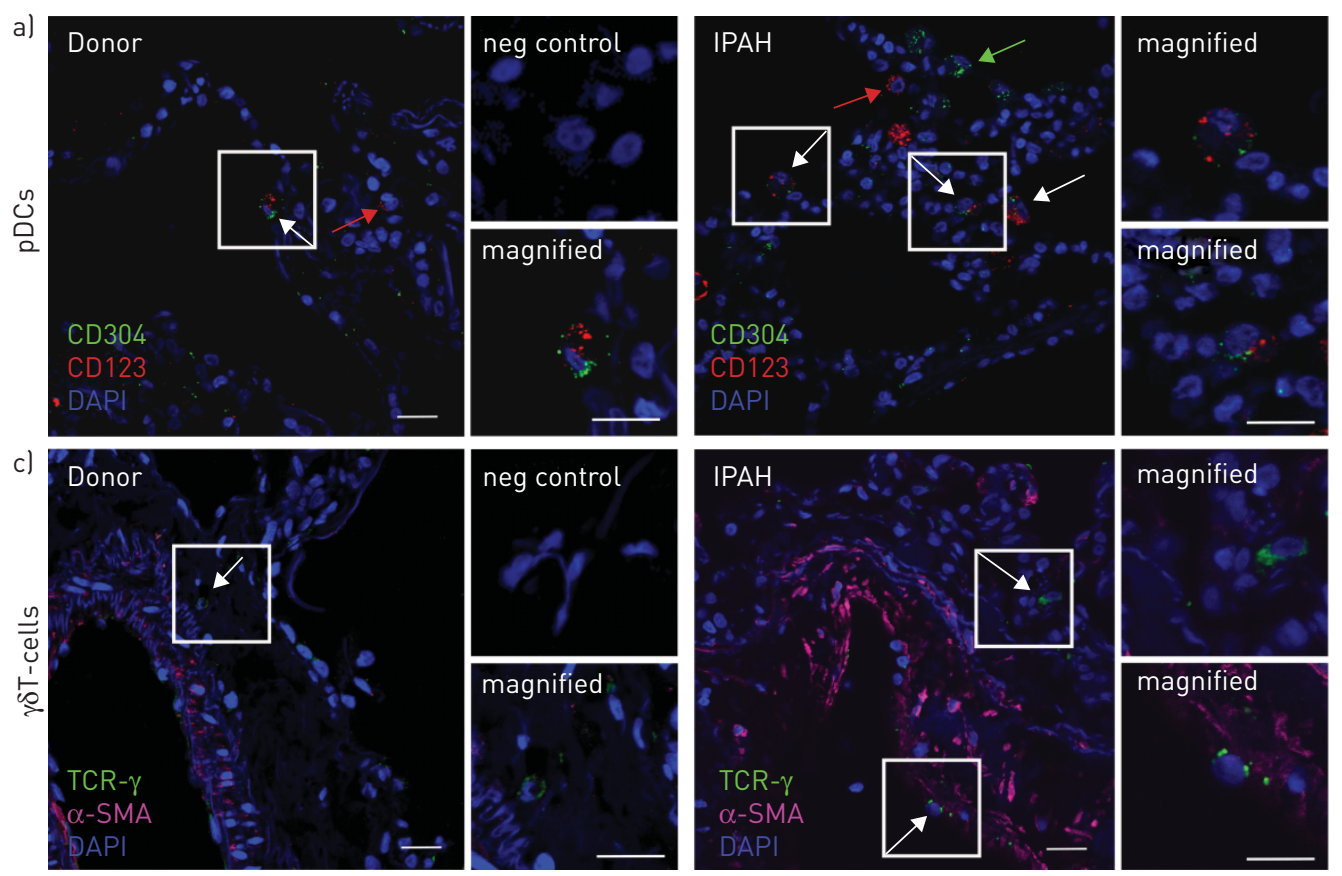
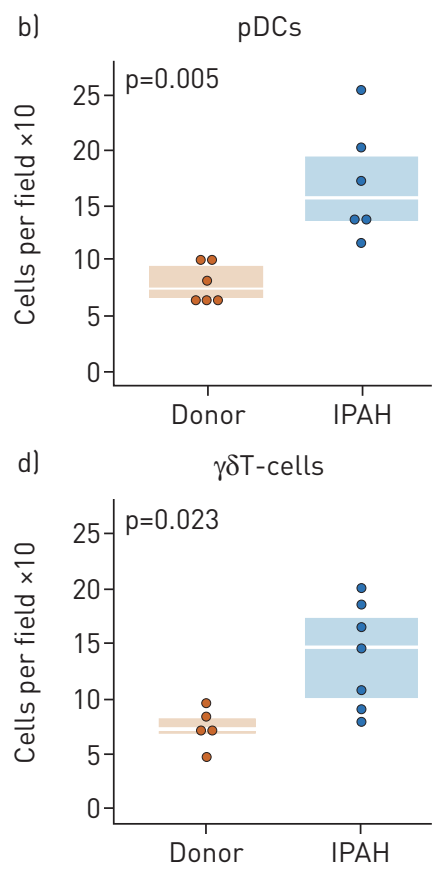

FIGURE 7 Presence and localisation of plasmacytoid dendritic cells (pDCs) and $\gamma \delta T$-cells in the lung. Immunofluorescence staining and quantification of idiopathic pulmonary arterial hypertension (IPAH) and donor lung tissue against a,b) CD123 (red) and CD304 (green) to detect pDCs (CD $123^{+} \mathrm{CD}_{304^{+}}$) and $\left.\mathrm{c}, \mathrm{d}\right) \mathrm{TCR} \gamma$ (green) to detect $\gamma \delta \mathrm{T}$-cells. Smooth muscle actin (SMA) staining is shown in purple; nuclear staining via DAPI (blue). Smaller panels represent magnified area as shown in larger panels. White arrows indicate cells of interest, red and green arrows indicate single positive cells. Neg control: staining performed in the absence of primary antibodies. Scale bars $=20 \mu \mathrm{m}$.

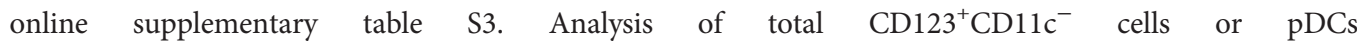
$\left(\mathrm{CD} 123^{+} \mathrm{CD} 11 \mathrm{c}^{-} \mathrm{CD} 304^{+} \mathrm{HLR}-\mathrm{DR}^{+}\right)$did not reveal any significant increases. Similarly, the other $\mathrm{CD} 123^{+}$ population, basophils $\left(\mathrm{CD} 123^{+} \mathrm{CD} 11 \mathrm{c}^{-} \mathrm{CD} 304^{-} \mathrm{FceRi}^{+}\right)$, were not elevated (figure 8 ). These results indicate that these populations are selectively increased in the lungs of $\mathrm{PAH}$ patients.

\section{Discussion}

In this study, we have created the most comprehensive picture of the immune cell populations within IPAH lungs to date. Using an unsupervised and unbiased approach we have evaluated the presence and abundance of 21 different inflammatory cell populations, thereby not only detailing the inflammatory landscape in healthy control lungs, but also that of IPAH patients. Previous studies that have examined the presence of inflammatory cells in the lungs of $\mathrm{PH}$ patients have predominantly relied on traditional scoring or immunohistochemistry analysis [1,4-6, 12, 21]. Although these techniques possess several strengths, such as the ability to quickly recognise architectural and structural disturbances, they are limited by the number of cell populations that can be analysed simultaneously. In contrast, flow cytometry permits the concurrent analysis of multiple independent cell populations using a large number of differentiation markers, including the multidimensional description of subpopulations and their activation states. Using this approach together with concomitant bioinformatical analysis we describe 1) a detailed picture of the immune phenotype in healthy lungs; 2) an altered immune cell signature in IPAH; and 3) novel regulated cell populations contributing to the immune disequilibrium in IPAH.

In IPAH patients, we not only observed a change in cell distribution (thus a different profile), but also increased abundance of inflammatory cells. As our analysis was restricted to IPAH and control samples we cannot ascertain whether this profile is specific for IPAH or may be shared with other pathologic conditions. Future studies should compare the findings with other patient groups, e.g. Eisenmenger's syndrome. However, even in such patients an inflammatory component cannot be excluded, as immune responses may also be involved in the response to increased flow and/or pressure. This is exemplified by studies using a rat model of $\mathrm{PH}$ due to left heart disease following supracoronary aortic banding. Here mast cells and their mediators were shown to be important for lung vascular remodelling and $\mathrm{PH}$ development [22].

Since vascular remodelling occurs in larger vessels as well as in small pulmonary arteries [16], we undertook another innovative approach and analysed the inflammatory profile in isolated larger 

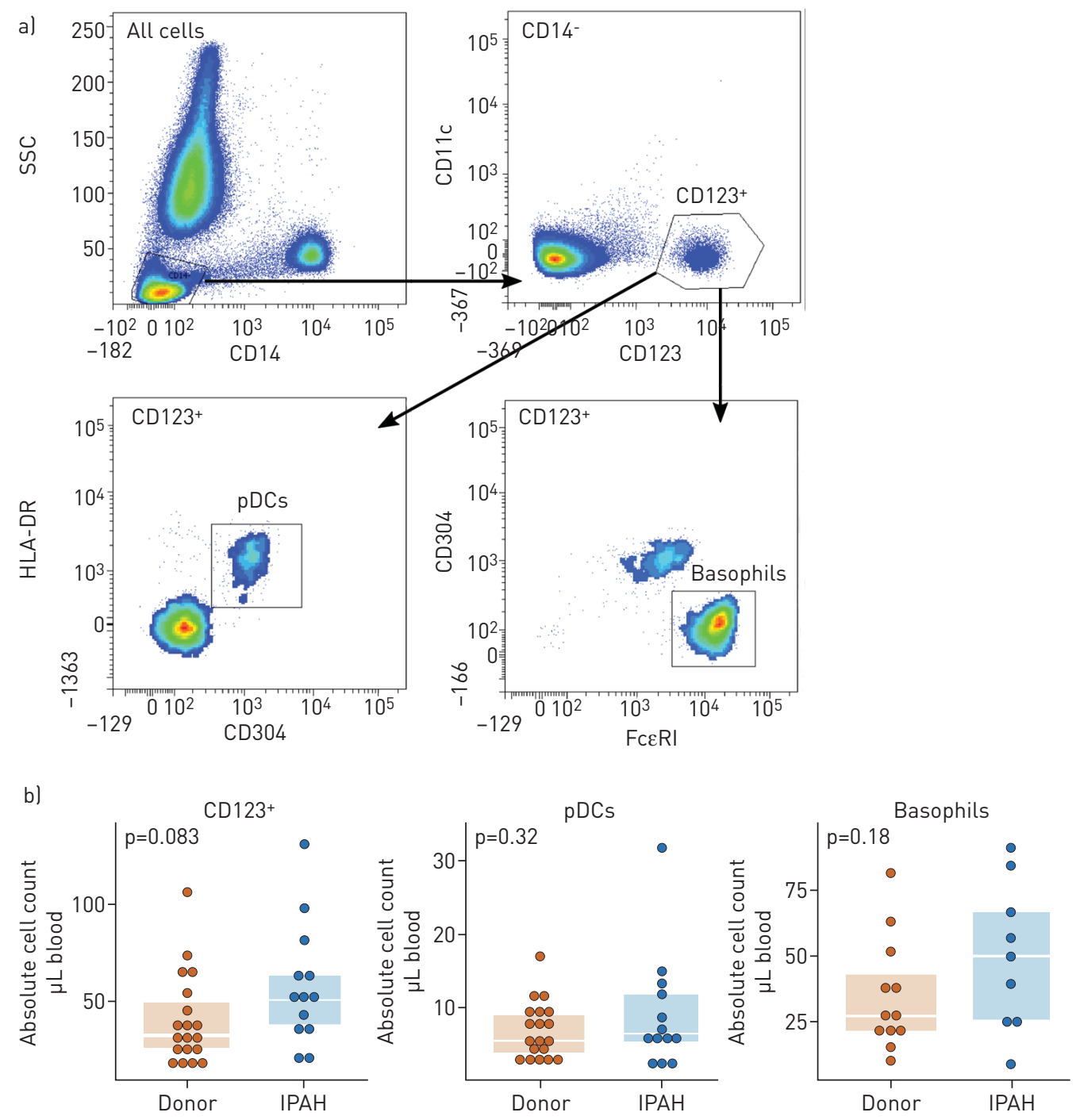

FIGURE 8 Idiopathic pulmonary arterial hypertension (IPAH) patients do not possess elevated plasmacytoid dendritic cell (pDC) numbers in the peripheral blood. a) Gating strategy for the flow cytometric quantification

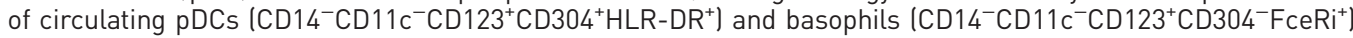
in peripheral blood. b) Scatterplot and boxplot overlays of quantified total CD11 $\mathrm{c}^{-} \mathrm{CD} 123^{+}$cells, pDCs and basophils. Boxplots show median and interquartile range.

pulmonary arteries $(>500 \mu \mathrm{m})$. Indeed, even in these pulmonary arteries changes in the inflammatory landscape were observed, which gives definitive evidence for the presence of inflammatory cells (including mast cells, activated macrophages and DCs) in larger remodelled vessels. The presence of activated macrophages within isolated pulmonary arteries supports the long standing premise of their involvement in $\mathrm{PH}[23,24]$.

Moving from the global inflammatory picture to specific cell types altered in IPAH, in our multidimensional flow cytometric characterisation, we observed increased mast cells and lymphocytes among others, which supports previous reports $[3-7,12,25]$ and demonstrates the validity of our approach. However, a few populations previously reported to be altered in $\mathrm{PH}$ were unchanged in our lung samples, e.g. B-cells or total macrophages [4, 24]. It is possible that differences in $\mathrm{PH}$ cohorts, markers used, or the use of enzymatic digestion could contribute to these differences. Unfortunately, our analysis panels could not differentiate between alveolar and interstitial macrophages, which require a number of additional markers including CD169 for accurate identification [13]. Future studies can make use of the continuing development of novel cell markers to specifically analyse these cell types.

Similar to mast cells, basophils (also increased in our IPAH cohort) are typical innate effector cells that can release a plethora of mediators such as interleukin (IL)-6, IL-13 or leukotriene B4, all implicated in 
$\mathrm{PH}$ pathogenesis [26-29], which in turn can activate and recruit T-cells [30]. In line with this we observed a strong increase in abundance of diverse T-cell subpopulations, namely $\mathrm{CD} 4^{+}, \mathrm{CD} 8^{+}$and $\gamma \delta \mathrm{T}$-cells in IPAH patients [25]. Interestingly, we have previously shown decreased numbers of circulating lymphocytes in IPAH, which indicates active recruitment to the lung [31]. In this study, we are the first to describe the increased presence of $\gamma \delta \mathrm{T}$-cells in IPAH. $\gamma \delta \mathrm{T}$-cells serve as a bridge between innate (e.g. NK cells and macrophages) and adaptive immune (e.g. B-cells and $\mathrm{CD} 4^{+} \mathrm{T}$-cells) responses [32]. In the skin, $\gamma \delta \mathrm{T}$-cells have important roles in tissue homeostasis and wound healing [33] by releasing insulin-like growth factor-1, which was previously shown to enhance smooth muscle cell proliferation [34].

A crucial cell type responsible for T-cell activation are DCs, which are ontologically derived from two distinct lineages, myeloid DCs $\left(\mathrm{CD} 11 \mathrm{c}^{+}\right.$and/or $\mathrm{CD}_{209^{+}}(\mathrm{DC}-\mathrm{SIGN})$ ) and pDCs $\left(\mathrm{CD} 123^{+} \mathrm{CD} 11 \mathrm{c}^{-}\right)$. Conclusive identification of DCs using only one marker is extremely challenging; thus, we additionally used HLA-DR (major histocompatibility complex class II) positivity and CD14-negativity to identify both $\mathrm{CD}_{209^{+}}$and CD11c ${ }^{+}$DCs. We observed an increased abundance of myeloid DCs, including CD209- DCs in the lungs and pulmonary arteries of IPAH patients, thereby expanding on previous observations $[5,12]$. In addition, we observed increased numbers and complexity of activated DCs and macrophages, as shown by CD1a (a transmembrane glycoprotein important in the presentation of lipid and glycolipid antigens) positivity in IPAH samples, which could further potentiate T-cell activation. The regulation of both DCs and T-cells indicate a strong adaptive immunity component underlying $\mathrm{PH}$ pathogenesis.

$\mathrm{PH}$ has been reported to possess autoimmune features $[5,35]$ and can develop in a variety of autoimmune diseases, such as systemic lupus erythematosus or systemic sclerosis [36, 37]. Similar to $\gamma \delta \mathrm{T}$-cells, pDCs have a key role in the regulation of autoimmunity and are at the crossroads of innate and adaptive immunity [38]. We here show for the first time elevated numbers of pDCs in the lungs of IPAH patients. Similarly to WANG et al. [39], we observed no changes in pDC numbers in the peripheral blood, which could indicate lung specific changes or may be due to differences in disease severity between explant and outpatient groups (New York Heart Association class III-IV versus II-III, respectively). pDCs are the most important producers of antiviral type-I interferons [40], which have been implicated in PH pathogenesis [41]. Therefore, pDCs might represent the missing link between the associations of $\mathrm{PH}$ with both autoimmunity and viral infections [42]. Alternatively, pDCs can also induce regulatory or anti-inflammatory responses [43]. In animal models, regulatory immune responses, e.g. via regulatory T-cells have been shown to be strongly protective of the $\mathrm{PH}$ phenotype [44]. It is therefore conceivable that the increased pDCs in the IPAH lungs may thus represent a mechanism to counter ongoing inflammatory processes.

The presence of inflammatory cells in control and IPAH samples highlights the importance of investigating how the immune response changes from a physiological to a maladaptive one. We can speculate that multiple changes accumulate until one molecule/cell becomes the proverbial straw which causes the remodelling cascade. Furthermore, it is likely that the global profile (the specific composition, distribution and interaction) and not only the increased abundancy of one specific cell type, is important for disease progression. Altogether, our data support the model of "smouldering inflammation" in PAH which was recently put forward by VOELKel et al. [45].

It is possible that several factors may influence the observed inflammatory cell profile, for example the BMPR2 status of these patients is unknown, so we cannot comment the impact this mutation could have. It is also possible that $\mathrm{PH}$ medication could alter the inflammatory signature, for example prostanoids have been shown to affect nuclear factor- $\mathrm{\kappa B}$ signalling [46], while phosphodiesterase-5 inhibitors can raise cGMP and nitric oxide levels which can have inflammatory dampening effects [47]. However, clinical evidence of anti-inflammatory effects of these medications in PH patients is lacking. Finally, our analysis is limited to transplanted end-stage lungs. Future studies are needed to address the mechanistic effects of the immune cells in IPAH. Along these lines, in several mouse model studies immune cells and their secreted mediators have been shown to give rise to pulmonary arterial remodelling and increased right ventricular pressure $[29,48,49]$.

Our use of flow cytometry to analyse inflammatory cell populations in the lungs of IPAH patients is novel and highlights the wealth of information that can be obtained by the simultaneous analysis of multiple cell populations. As more research groups incorporate these techniques into their analysis of inflammatory populations, it will further increase the resolution of the inflammatory landscape of pulmonary hypertension and potentially even stratify patients according to their inflammatory status.

\section{Acknowledgements}

We would like to thank Sabrina Reinisch, Nina Treitler, Lisa Oberreiter and Eva Grasmann (Ludwig Boltzmann Institute for Lung Vascular Research, Graz, Austria) for their excellent technical assistance and Jochen Wilhelm (Universities of Giessen and Marburg Lung Center, Giessen, Germany) for his guidance with computational analysis. 


\section{References}

1 Stacher E, Graham BB, Hunt JM, et al. Modern age pathology of pulmonary arterial hypertension. Am J Respir Crit Care Med 2012; 186: 261-272.

2 Nicolls MR, Voelkel NF. The roles of immunity in the prevention and evolution of pulmonary arterial hypertension. Am J Respir Crit Care Med 2017; 195: 1292-1299.

3 Heath D. Pulmonary hypertension in pulmonary parenchymal disease. Cardiovasc Clin 1972; 4: 79-96.

4 Tuder RM, Groves B, Badesch DB, et al. Exuberant endothelial cell growth and elements of inflammation are present in plexiform lesions of pulmonary hypertension. Am J Pathol 1994; 144: 275-285.

5 Perros F, Dorfmüller P, Souza R, et al. Dendritic cell recruitment in lesions of human and experimental pulmonary hypertension. Eur Respir J 2007; 29: 462-468.

6 Montani D, Perros F, Gambaryan N, et al. C-kit-positive cells accumulate in remodeled vessels of idiopathic pulmonary arterial hypertension. Am J Respir Crit Care Med 2011; 184: 116-123.

7 Kwapiszewska G, Markart P, Dahal BK, et al. PAR-2 inhibition reverses experimental pulmonary hypertension. Circ Res 2012; 110: 1179-1191.

8 Balabanian K, Foussat A, Dorfmüller P, et al. CX(3)C chemokine fractalkine in pulmonary arterial hypertension. Am J Respir Crit Care Med 2002; 165: 1419-1425.

9 Dorfmüller P, Zarka V, Durand-Gasselin I, et al. Chemokine RANTES in severe pulmonary arterial hypertension. Am J Respir Crit Care Med 2002; 165: 534-539.

10 Itoh $\mathrm{T}$, Nagaya $\mathrm{N}$, Ishibashi-Ueda $\mathrm{H}$, et al. Increased plasma monocyte chemoattractant protein-1 level in idiopathic pulmonary arterial hypertension. Respirology 2006; 11: 158-163.

11 Zabini D, Crnkovic S, Xu H, et al. High-mobility group box-1 induces vascular remodelling processes via c-Jun activation. J Cell Mol Med 2015; 19: 1151-1161.

12 Savai R, Pullamsetti SS, Kolbe J, et al. Immune and inflammatory cell involvement in the pathology of idiopathic pulmonary arterial hypertension. Am J Respir Crit Care Med 2012; 186: 897-908.

13 Bharat A, Bhorade SM, Morales-Nebreda L, et al. Flow cytometry reveals similarities between lung macrophages in humans and mice. Am J Respir Cell Mol Biol 2016; 54: 147-149.

14 Yu YR, Hotten DF, Malakhau Y, et al. Flow cytometric analysis of myeloid cells in human blood, bronchoalveolar lavage, and lung tissues. Am J Respir Cell Mol Biol 2016; 54: 13-24.

15 Desch AN, Gibbings SL, Goyal R, et al. Flow cytometric analysis of mononuclear phagocytes in nondiseased human lung and lung-draining lymph nodes. Am J Respir Crit Care Med 2016; 193: 614-626.

16 Hoffmann J, Wilhelm J, Marsh LM, et al. Distinct differences in gene expression patterns in pulmonary arteries of patients with chronic obstructive pulmonary disease and idiopathic pulmonary fibrosis with pulmonary hypertension. Am J Respir Crit Care Med 2014; 190: 98-111.

17 Slama A, Ghanim B, Klikovits T, et al. Lobar lung transplantation - is it comparable with standard lung transplantation? Transpl Int 2014; 27: 909-916.

18 van der Maaten L, Hinton G. Visualizing data using t-SNE. J Mach Learn Res 2008; 9: 2579-2605.

19 Kwapiszewska G, Chwalek K, Marsh LM, et al. BDNF/TrkB signaling augments smooth muscle cell proliferation in pulmonary hypertension. Am J Pathol 2012; 181: 2018-2029.

20 Saeys Y, Gassen SV, Lambrecht BN. Computational flow cytometry: helping to make sense of high-dimensional immunology data. Nat Rev Immunol 2016; 16: 449-462.

21 Caslin AW, Heath D, Madden B, et al. The histopathology of 36 cases of plexogenic pulmonary arteriopathy. Histopathology 1990; 16: 9-19.

22 Hoffmann J, Yin J, Kukucka M, et al. Mast cells promote lung vascular remodelling in pulmonary hypertension. Eur Respir J 2011; 37: 1400-1410.

23 Frid MG, Brunetti JA, Burke DL, et al. Hypoxia-induced pulmonary vascular remodeling requires recruitment of circulating mesenchymal precursors of a monocyte/macrophage lineage. Am J Pathol 2006; 168: 659-669.

24 El Kasmi KC, Pugliese SC, Riddle SR, et al. Adventitial fibroblasts induce a distinct proinflammatory/profibrotic macrophage phenotype in pulmonary hypertension. J Immunol 2014; 193: 597-609.

25 Austin ED, Rock MT, Mosse CA, et al. T lymphocyte subset abnormalities in the blood and lung in pulmonary arterial hypertension. Respir Med 2010; 104: 454-462.

26 Humbert M, Monti G, Brenot F, et al. Increased interleukin-1 and interleukin-6 serum concentrations in severe primary pulmonary hypertension. Am J Respir Crit Care Med 1995; 151: 1628-1631.

27 Hecker M, Zaslona Z, Kwapiszewska G, et al. Dysregulation of the IL-13 receptor system: a novel pathomechanism in pulmonary arterial hypertension. Am J Respir Crit Care Med 2010; 182: 805-818.

28 Tian W, Jiang X, Tamosiuniene R, et al. Blocking macrophage leukotriene B4 prevents endothelial injury and reverses pulmonary hypertension. Sci Transl Med 2013; 5: 200ra117.

29 Park SH, Chen WC, Esmaeil N, et al. Interleukin 13- and interleukin 17A-induced pulmonary hypertension phenotype due to inhalation of antigen and fine particles from air pollution. Pulm Circ 2014; 4: 654-668.

30 Sokol CL, Barton GM, Farr AG, et al. A mechanism for the initiation of allergen-induced T helper type 2 responses. Nat Immunol 2008; 9: 310-318.

31 Foris V, Kovacs G, Marsh LM, et al. CD133 ${ }^{+}$cells in pulmonary arterial hypertension. Eur Respir J 2016; 48: 459-469.

32 Fay NS, Larson EC, Jameson JM. Chronic inflammation and $\delta \gamma$ T cells. Front Immunol 2016; 7: 210.

33 Jameson J, Havran WL. Skin $\delta \gamma$ T-cell functions in homeostasis and wound healing. Immunol Rev 2007; 215: $114-122$.

34 Dempsey EC, Badesch DB, Dobyns EL, et al. Enhanced growth capacity of neonatal pulmonary artery smooth muscle cells in vitro: dependence on cell size, time from birth, insulin-like growth factor I, and auto-activation of protein kinase C. J Cell Physiol 1994; 160: 469-481.

35 Colvin KL, Cripe PJ, Ivy DD, et al. Bronchus-associated lymphoid tissue in pulmonary hypertension produces pathologic autoantibodies. Am J Respir Crit Care Med 2013; 188: 1126-1136.

36 Proudman SM, Stevens WM, Sahhar J, et al. Pulmonary arterial hypertension in systemic sclerosis: the need for early detection and treatment. Intern Med J 2007; 37: 485-494.

37 Trad S, Amoura Z, Beigelman C, et al. Pulmonary arterial hypertension is a major mortality factor in diffuse systemic sclerosis, independent of interstitial lung disease. Arthritis Rheum 2006; 54: 184-191. 
Colonna M, Trinchieri G, Liu YJ. Plasmacytoid dendritic cells in immunity. Nat Immunol 2004; 5: 1219-1226.

Wang W, Yan H, Zhu W, et al. Impairment of monocyte-derived dendritic cells in idiopathic pulmonary arterial hypertension. J Clin Immunol 2009; 29: 705-713. is triggered by viruses, but the level of production is controlled by distinct cytokines. J Interferon Cytokine Res 2002; 22: 653-659.

41 George PM, Oliver E, Dorfmuller P, et al. Evidence for the involvement of type I interferon in pulmonary arterial hypertension. Circ Res 2014; 114: 677-688.

42 Petitpretz P, Brenot F, Azarian R, et al. Pulmonary hypertension in patients with human immunodeficiency virus infection. Comparison with primary pulmonary hypertension. Circulation 1994; 89: 2722-2727.

43 Audiger C, Rahman MJ, Yun TJ, et al. The importance of dendritic cells in maintaining immune tolerance. J Immunol 2017; 198: 2223-2231.

44 Tamosiuniene R, Tian W, Dhillon G, et al. Regulatory $\mathrm{T}$ cells limit vascular endothelial injury and prevent pulmonary hypertension. Circ Res 2011; 109: 867-879.

45 Voelkel NF, Tamosiuniene R, Nicolls MR. Challenges and opportunities in treating inflammation associated with pulmonary hypertension. Expert Rev Cardiovasc Ther 2016; 14: 939-951.

46 Birukova AA, Wu T, Tian Y, et al. Iloprost improves endothelial barrier function in lipopolysaccharide-induced lung injury. Eur Respir J 2013; 41: 165-176.

47 Peixoto CA, Gomes FO. The role of phosphodiesterase-5 inhibitors in prostatic inflammation: a review. J Inflamm 2015; 12: 54

48 Daley E, Emson C, Guignabert C, et al. Pulmonary arterial remodeling induced by a Th2 immune response. J Exp Med 2008; 205: 361-372.

49 Kumar R, Mickael C, Chabon J, et al. The causal role of IL-4 and IL-13 in Schistosoma mansoni pulmonary hypertension. Am J Respir Crit Care Med 2015; 192: 998-1008. 